\title{
Classification of Several WSN Routing Protocols
}

\author{
Minakshi Roy \\ Assistant Professor, \\ Dept.of Computer science \& \\ Engg \\ Sikkim Manipal Institute of \\ Technology \\ Majhitar, Sikkim
}

\author{
Debjani Bhowmik \\ Assistant Professor, \\ Dept. of Computer Science \\ Engg. \\ The ICFAI University, Tripura
}

\author{
Chitrapriya N. \\ Assistant Professor, \\ Dept.of Computer science \& \\ Engg \\ Sikkim Manipal Institute of \\ Technology \\ Majhitar, Sikkim
}

\begin{abstract}
The Wireless sensor network (WSN) is the renowned name in the area of research due to the large possibilities of sensor networks to provide many fields such as military applications, health monitoring, environmental monitoring etc. The recent WSN technology has given the receptiveness of tiny and cheaper cost sensor nodes with capability of sensing various types of rural, dangerous environmental conditions, integrated circuit technologies, distributed signal processing, data processing, wireless communications, Ad-hoc networking routing protocols, micro electro-mechanical systems technology, , nano technology, microprocessor hardware, and embedded systems have made the concept of Wireless Sensor Network. Sensor network nodes are restricted with respect to energy supplying, limited computational power and communication bandwidth. So the main aim is to design effective and energy based protocol to increase the network lifetime. In WSN, the sensor nodes have a little transmission range, their processing and storage capacity and aslo their energy resources are limited. In this paper, we have given a survey of routing protocols for Wireless Sensor Network with their advantages and limitations and also analyzed the design issues of sensor networks. These classifications of protocols are done based on the energy efficiency, data-centric, hierarchical and location based depending on the network structure. Some of these criteria are further detailed classified. We have also emphasized some extending characteristics of some protocols. The design challenges of WSN are also discussed with its application area.
\end{abstract}

\section{Keywords}

Wireless sensor network, Routing, classification, Application.

\section{INTRODUCTION}

Wireless sensor network (WSN) is a technologies widely considered for the twenty-first century. A WSN typically consists of a low-cost, low-power, and multifunctional wireless sensor nodes, with sensing, wireless communications and computation capabilities. Communication in WSN is possible over short distance via a wireless medium and collaborates to accomplish a common task. It is widely used in environment monitoring, military surveillance, and industrial process control. The capability of each individual sensor node is limited, the aggregate power of the entire network is sufficient for the required mission. Sensor nodes are battery-powered and are expected to operate without attendance for a relatively long period of time. Sometimes, it is very difficult and even impossible to change or recharge batteries for the sensor nodes.
In traditional routing protocols have various shortcomings when applied to WSNs, due to the energy-constrained nature of such networks. Furthermore, these inconveniences are highlighted when the number of nodes in the network increases. Some research activities explore and overcome the constraints of WSNs and solve design and application issues. Sensor networks is an emerging and promising tool for monitoring the physical worlds, utilizing self-organizing networks of battery-powered, process and communicate. In sensor networks, energy is treated as a critical resource. [2]

The WSN is built by using several nodes, from a few to several hundreds or even thousands, where each node is connected to one or with several sensors. Every sensor network node has several parts: it has a radio transceiver with an antenna connected internally or externally, a microcontroller for interfacing with the sensors and an energy source. A constraint on size and cost of a sensor node corresponds to a constraint on energy, memory, computational speed and communications bandwidth. So, to minimize any energy consumption, routing techniques were used in a WSN network. A sensor network [2] is a network of many tiny disposable low power devices, called nodes, which are distributed partially in order to perform an applicationoriented task globally.

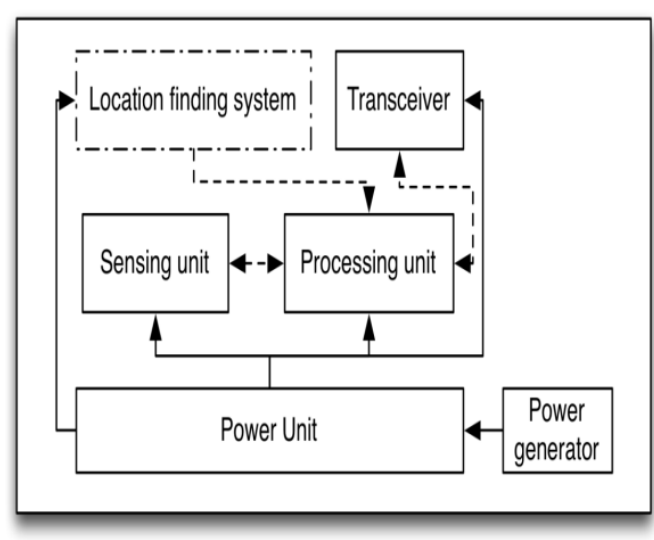

Fig 1. Architecture of a sensor node

A network is formed from the nodes by communicating with each other directly or through other nodes as indirectly. A single node or many nodes among the network will perform as sink having an ability to communicate with users directly or by using existing wired networks. The fundamental component of a network is sensor which is an essential part responsible for monitoring real world physical conditions such as sound, vibration, humidity, pressure, motion, intensity pollutants, temperature etc. at separate locations. A small sensor node includes sensing on board processor for data 
processing, and communicating components. Fig 1 shows the architecture of a sensor node, where each node typically consists of the four components namely a sensor unit, power unit, central processing unit (CPU) and communication and each unit are assigned with respective tasks.A sensor unit includes a sensor and Analog to Digital Converter (ADC). This unit is responsible for collecting information requested by ADC and returning back the analog data. The task of ADC is to inform the CPU what the sensor unit has sensed and also tells the sensor unit what is supposed to be done. The purpose of communication unit is to receive command or query from CPU and transmit it to the outside world. Basically CPU is the most complex unit. It will interpret the queries for ADC and processes any received data. It can control and monitors power. Also it computes the next hop and inform to the sink. The work of a power unit is to supply power to all the other component units.

All nodes may have two other optional components apart from the basic unit such as Mobilizer (to move sensor nodes when it is required to carry out the assigned tasks) and location finding system (the knowledge of location with high accuracy). Sensor node can performed simple computations transmit only the required and partially processed data rather than sending the whole raw data to the nodes. It not only gathers useful information like temperature, sound, light but also plays a role in communicating through wireless channels in the router under the constraints of battery. The limitation of a sensor nodes are with respect to computational capacity, in energy supply and communication bandwidth. But an ideal wireless sensor is scalable, consume less power, fault tolerance, they are smart and programmable. Apart from these they are capable of fast data acquisition, efficient, reliable and accurate over long term. And lastly, the main advantage is low cost and easy or no maintenance.

The main tasks of a WSN are to: Calculate the given location's physical value, determine the estimate parameters of the detected event and occurrence of events of interest, Trace an object and divide any object which was detected.

The important requirements of a WSN are: (i) use of a large number of sensors, (ii) attachment of stationary sensors, (iii) low energy consumption, (iv) self organization capability, (v) collaborative signal processing, and (vi) querying ability.

In this paper routing protocols of wireless sensor network are described and classified. The second section of the paper explains the characteristics of the network and its design objectives. Third section contains applications of sensor networks. In Sections 4, various routing protocols are discussed and classified. In Section 5, routing issues are described. Finally, Section 6 concludes the survey of sensor networks.

\section{NETWORK CHARACTERISTICS AND DESIGN OBJECTIVES:}

\subsection{Characteristics of WSN:}

Network Architecture: Sensor network consists of three main components. These are the sink, monitored events and sensor nodes. Routing messages from moving node more challenging since route stability is more important factor, in addition to energy , bandwidth etc. [7]

Dense sensor node deployment: Sensor nodes are usually densely deployed in the wireless sensor network area. [1]

Node capabilities: All sensor nodes are assumed to be homogenous, having equal capacity in terms of computation, communication and power. Set of sensors raises multiple technical issues related to data routing. [7]

Battery-powered sensor nodes: Sensor nodes are usually powered by battery and are deployed in a harsh environment where it is very difficult to change or recharge the batteries. [1]

Severe energy, computation, and storage constraints: Sensors nodes are having highly limited energy, computation, and storage capabilities.

Self-configurable: Sensor nodes are usually randomly deployed and autonomously configure themselves into a communication network.

Unreliable sensor nodes: Since sensor nodes are prone to physical damages or failures due to its deployment in harsh or hostile environment.

Data redundancy: In most sensor network application, sensor nodes are densely deployed in a region of interest and collaborate to accomplish a common sensing task. Thus, the data sensed by multiple sensor nodes typically have a certain level of correlation or redundancy.

Application specific: A sensor network is usually designed and deployed for a specific application. The design requirements of a sensor network change with its application.

Many-to-one traffic pattern: In most sensor network applications, the data sensed by sensor nodes flow from multiple source sensor nodes to a particular sink, exhibiting a many-to-one traffic pattern.

Frequent topology change: Network topology changes frequently due to the node failures, damage, addition, energy depletion, or channel fading. [1]

Data Latency and Over Head :

These are considered as the important factors that influence routing protocol design. Data aggregation and multi hop relays cause data latency. Some routing protocols create excessive overheads to implement their algorithms, which are not suitable for energy constrained network.[7]

\subsection{Network design objectives}

Sensor networks are designed for several applications and they also have several requirements for such applications. So, several parts of design objectives are considered during the design of WSN. Following are such essential design objectives:

Tiny Node size: As the node size is small therefore it facilitates to deployment of node in the harsh environment. It will also limit the cost and power consumption of sensor nodes.

Minimum node cost: As plenty of sensor nodes are usually deployed in a hostile environment in large numbers and it does not have the capability of reuse, so minimizing cost of sensor nodes is essential. Finally it it will reduce the cost of the whole network.

Low power consumption: Since sensor nodes are powered by battery and it is often very difficult or even impossible to charge or recharge their batteries, it is crucial to reduce the power consumption of sensor nodes so that the lifetime of the sensor nodes, as well as the whole network is prolonged.

Scalability: As the sensor nodes are available in sensor networks in the order of tens, or multipliers of ten, for eg. 
hundreds, thousands etc, the design of network protocols should be scalable to the different sizes of the network.

Reliability: It should give error control and correction mechanisms to ensure error free or data delivery over traffic some, error-prone, and busy wireless channels.

Self-configurability: It is an important characteristic of sensor networks, once nodes are deployed, they should be able to autonomatically construct themselves into a communication network and reconfigure their connectivity in the event of topology changes and node failures.

Adaptability: In sensor networks, a node may fail, join, or move, which would result in changes in node density and network topology. Thus, network protocols designed for sensor networks should be adaptive to such density and topology changes.

Channel utilization: Since sensor networks have limited bandwidth resources, communication protocols designed for sensor networks should efficiently make use of the bandwidth to improve channel utilization.

Fault tolerance: Sensor nodes are prone to failures due to harsh deployment environments and unattended operations. Thus, sensor nodes should be fault tolerant and have the abilities of self testing, self-calibrating, self-repairing, and self-recovering.

Security: A sensor network should introduce effective security mechanisms to prevent the data information in the network or a sensor node from unauthorized access or malicious attacks.

QoS support: In sensor networks, different applications may have different quality-of-service (QoS) requirements in terms of delivery latency and packet loss. Thus, network protocol design should consider the QoS requirements of specific applications. [1]

\section{APPLICATIONS OF SENSOR NETWORKS}

Wireless sensor network is having a wild field of application. The sensor networks can be used in remote areas for disaster Relief, military, emergency rescue operation and habitat Monitoring, environmental monitoring, health Care, home networks, radiological, nuclear, detecting chemical, biological, explosive material and many more.

Emergency situations: Heavy Fire/water detectors, disaster management, risky chemical level and fires.

Military Service: In military sensor network is a most useful area these are used for the following situation like sudden awareness, detection of enemy unit movements on land, sensing intruders on basis and sea and battle field surveillances.

Health: Sensors for ECG (electrocardiogram), blood flow, blood pressure, pulse oxymeter, breathing rate, monitoring people's location and health condition and oxygen measurement.

Home networks: Household gadgets, person locator, apparatus, region awareness.

Automatic pressure tracking: Active mobile facility, integrated vehicle tracking.
Environment: Physical monitoring of aqua and soil, habitual monitoring, Observation of organic life and manufactured systems.

Industrial Factory: Industrial automation, process control, control and monitoring of industrial equipment. [2]

\section{WSN ROUTING PROTOCOLS}

WSN Routing Protocols can be divided into protocols like Location based protocol, Data-centric protocol, Hierarchical Protocol, Mobility based protocol, Multipath based protocol, QoS based protocol and Operational based routing.

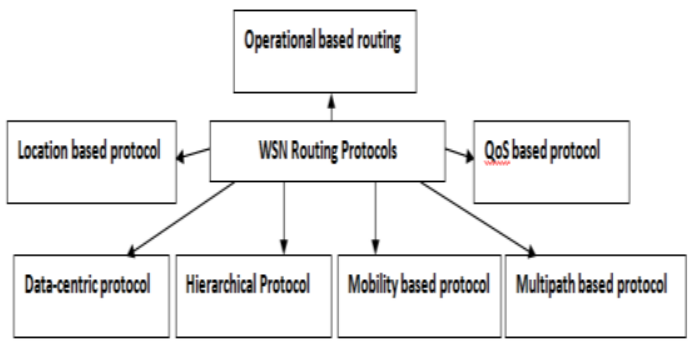

Fig 2. Several WSN routing protocols

In Location-based Protocols locations are sensed by the sensor nodes. For the sensor networks, sensing locations are one of the most important task. Using these nodes, the distance between two particular nodes can be calculated and from this distance energy consumption can be estimated. Some of the protocols of this types are- MECN, GAF, Span, TBF, BVGF, GeRaF, SMECN, GEAR. These types of protocols use location to guide routing discovery. Data forwarding, enabling directional transmission of the information flooding in the network is also another important task of location based protocols.

Through Data centric protocols data is sent from source to sink. When the source sensors send their data to sink intermediate sensor perform some form of aggregation on the data originating from multiple source sensors and send the aggregated data toward the sink. By using this protocol energy is saved because of less transmission required to send the data from the source to sink

Hierarchical clustering is an important aspect in WSN. Hierarchical protocol is used by the sensors to report their sensed data to the sink. It is a energy efficient communication protocol. In this layered protocols a network is composed of several cluster of sensors. Each cluster is managed by a special node called cluster head, which is responsible for coordinating the data transmission activities of all sensors in its clusters.

Mobility is one of the important aspects of WSNs. Data originated from source sensors must reach toward mobile sinks through the mobility based protocols. Mobility is one of the most important aspects for these routing protocols.

Single path routing approach is unable to provide good data rate transmission in wireless sensors networks as the capacity is limited. But multipath routing approach is broadly utilized is the important approach as solution. Multipath based protocol provides multipath routing facility.

Quality of service requirement is required to minimize energy consumption and delay in communication. To increase the reliability in routing quality of service is an important aspect. 
These types of protocols are classified into SPEED, Energyaware routing, SAR.

When WSNs applications are categorised according to their functionalities we get operational based routing .Here protocols are classified according to their operations to meet these functionalities .Their classification achieve optimal performance to save the scarce resources of the network. Operational based routing protocol provides operational based routing. Operational based routing protocols are of the types like

- Data Delivery Model: Here data is collected by the node in any data delivery model.

- Continuous Model: Data of same size is generally collected by each sensor node and by multi hop connection data is transferred periodically to the base station. Examples: MCFA, SPEED, LEACH, GAF, TEEN, PEGASIS, CADR, SAR, MECN, SMECN.

- Query-Driven Model: Here query is generated by the sink and then data is transmitted. All the related information is sensed by the node and send back to sink.

- Event-Driven Model: In this model the transmission of data is triggered only is the event of interest occurs. The observer is interested only in the occurrence of a specific phenomenon or set of phenomena.

- Hybrid Model: This model is using a combination of continuous, event-driven and query-driven data delivery.

\section{ROUTING ISSUES}

To design routing protocols with sensor network some constraints exist. Restriction of several network resources affect WSNs, Constraints may be bandwidth, storage, CPU and energy.

WSNs are designed so that it should be as energy efficient as possible to extend their lifetime, It will prolongs the networks lifetime, Energy is the most important issue for network designers. WSNs protocol can manage location of the sensors. Sensors are equipped with GPS receivers. Sensor's processing and storage capabilities are limited and performed computational functionalities also limited. Hardware limitations become the constraints for software development. Sensors may not necessarily have the same capabilities in terms of energy, processing, sensing, and Particularly communication for different size of the networks. So protocols should be able to scale with the network size. Some sensor nodes should not fail or be blocked due to lack of power or physical damage or environmental interference. The failure of sensor nodes should not affect the overall task of the sensor network. This is the reliability issue. : Sensor networks have a wide range of diverse applications. No network protocol can meet the requirements of all applications. Therefore, routing protocols should guarantee data delivery and its accuracy so that the sink can gather the required knowledge about the physical phenomenon on time. Since sensor nodes may generate significant redundant data, similar packets from multiple nodes can be aggregated so that the number of transmissions is reduced. Data aggregation technique has been used to achieve energy efficiency and data transfer optimization in a number of routing protocols.

\section{CONCLUSION AND FUTURE WORK}

The main challenge in the design of routing protocols is energy efficiency. It is due to the lack of energy resources. The ultimate motto of designing and developing these routing protocols is to increase active session of the sensor network. Here this paper, we have classified the routing protocols into seven main categories. However, realization of sensor node satisfies the constraints with the factors such as fault tolerance, scalability, cost, hardware, topology change, environment, power consumption etc. In this paper, we have described important design issues of routing protocols for sensor networks and also classified the existing routing protocols. We have observed that it quite impossible to design and develop a routing algorithm which will have good performance with all constraints and for all applications. We still have many issues to be resolved although so many protocols exist.

\section{REFERENCES}

[1] Shio kumar Singh, MP Singh, and D K Singh, 2010 Routing protocols in wireless sensor networks - a survey. International journal of computer science \& engineering survey (ijcses) vol.1, no.2, november.

[2] Rajashree.v.biradar, V.C Patil, Dr. S. R Sawant, Dr. R. $\mathrm{R}$ Mudholkar Classification and comparison of routing protocols in wireless sensor networks. special issue on ubiquitous computing security systems, ubicc journal volume 4

[3] Neha Rathi, Jyoti Saraswat and Partha Pratim Bhattacharya, 2012 A Review on routing protocols for application in wireless sensor networks. International journal of distributed and parallel systems (ijdps) vol.3, no.5, september.

[4] Manal Abdullah, Aisha Ehsan, 2014 Routing protocols for wireless sensor networks: classifications and challenges. Quest journals journal of electronics and communication engineering research volume $2 \sim$ issue 2 pp: 05-15 issn(online) : 2321-5941.

[5] Mark A. Perillo and Wendi B. Heinzelman. Wireless sensor network protocols. Department of Electrical and Computer Engineering, University of Rochester, Rochester, NY, USA

[6] Navdeep Kaur, Deepika Sharma and Prabhdeep Singh, 2013. Classification of Hierarchical routing protocols in ireless sensor network: a survey. International journal of p2p network trends and technology- volume3issue 1.

[7] Ms. Parul Tyagi, Ms. Surbhi Jain 2012 Comparative study of routing protocols in wireless sensor network, volume 2, issue 9, September issn: 2277 128x.

[8] Abdul ganikhan : 2012 A comparative analysis, routing protocols for wireless sensor networks. Ijrim volume 2, issue 2 ,issn 2231-4334 .

[9] Wendi RabinerHeinzelman, AnanthaChandrakasan, and Hari Balakrishnan. 2000 :Energy-Efficient Communication Protocol for Wireless Microsensor Networks. Proceedings of the 33rd Hawaii International Conference on System Sciences.

[10] Yun li, nan yu1, weiyizhang, weiliangzhao , xiaohu you, mahmouddaneshmand 2011 Enhancing the performance of leach protocol in wireless sensor networks",Ieeeinfocom workshop on $\mathrm{m} 2 \mathrm{mcn}$.

[11] Harneetkour, ajay k. Sharma 2010 Hybrid energy efficient distributed protocol for heterogeneous wireless sensor network.International journal of computer applications $(0975-8887)$ volume 4 - no.6. 
International Journal of Computer Applications (0975 - 8887)

Volume 158 - No 8, January 2017

[12] Taheri,H.;Neamatollahi,P,Naghibzadeh, M,Yaghmaee, M. 2010 Improving on HEED protocol of wireless sensor networks using non probabilistic approach and fuzzy logic (HEED-NPF).Telecommunications (IST), 2010 5th International Symposium on , vol., no., pp.193198.

[13] Stephanie lindsey, cauligi s. Raghavendra :Pegasis: power-efficient gathering in sensor Information
systems.The Aerospace Corporation P.O. Box 92957 Los Angeles, CA 90009-2957.

[14] Indu Shukla: Power efficient gathering in sensor information system (pegasis protocol).

[15] Debnath Bhattacharyya, Tai-hoon Kim and Subhajit Pal: 2010 A Comparative Study of Wireless Sensor Networks and Their Routing Protocols.ISSN 1424-8220. 